\title{
Impact of parity on ewe vaginal mechanical properties relative to the nonhuman primate and rodent
}

\author{
Katrina M. Knight ${ }^{1}$, Pamela A. Moalli ${ }^{1,2}$, Alexis Nolfi ${ }^{2}$, Stacy Palcsey ${ }^{2}$, William R. Barone ${ }^{1}$, \\ and Steven D. Abramowitch ${ }^{1,2,3}$ \\ ${ }^{1}$ Department of Bioengineering, Musculoskeletal Research Center, University of Pittsburgh, \\ Pittsburgh, PA, USA \\ ${ }^{2}$ Magee-Womens Research Institute, Department of Obstetrics and Gynecology, University of \\ Pittsburgh, Pittsburgh, PA, USA \\ ${ }^{3}$ Musculoskeletal Research Center, 405 Center for Bioengineering, 300 Technology Drive, \\ Pittsburgh, PA 15219, USA
}

\begin{abstract}
Introduction and hypothesis-Parity is the leading risk factor for the development of pelvic organ prolapse. To assess the impact of pregnancy and delivery on vaginal tissue, researchers commonly use nonhuman primate (NHP) and rodent models. The purpose of this study was to evaluate the ewe as an alternative model by investigating the impact of parity on the ewe vaginal mechanical properties and collagen structure.
\end{abstract}

\begin{abstract}
Methods-Mechanical properties of 15 nulliparous and parous ewe vaginas were determined via uniaxial tensile tests. Collagen content was determined by hydroxyproline assay and collagen fiber thickness was analyzed using picrosirius red staining. Outcome measures were compared using Independent samples $t$ or Mann-Whitney $U$ tests. ANOVA (Gabriel's pairwise post-hoc test) or the Welch Alternative for the F-ratio (Games Howell post-hoc test) was used to compare data with previously published NHP and rodent data.
\end{abstract}

Results-Vaginal tissue from the nulliparous ewe had a higher tangent modulus and tensile strength compared with the parous ewe $(p<0.025)$. The parous ewe vagina elongated $42 \%$ more than the nulliparous ewe vagina $(p=0.015)$. No significant differences were observed in collagen structure among ewe vaginas. The tangent modulus of the nulliparous ewe vagina was not different from that of the NHP or rodent $(p=0.290)$. Additionally, the tangent moduli of the parous ewe and NHP vaginas did not differ $(p=0.773)$.

Correspondence to: Steven D. Abramowitch.

This article was presented at the American Society of Mechanical Engineers 2013 Summer Bioengineering Conference, Sunriver, OR, USA, 26-29 June 2013

Electronic supplementary material The online version of this article (doi:10.1007/s00192-016-2963-2) contains supplementary material, which is available to authorized users

Conflicts of interest K.M. Knight, A. Nolfi, S. Palcsey, and W. Barone have no conflicts of interest to disclose. P.A. Moalli received a research grant from the National Institutes of Health, R01 HD-061811, and has a cooperative research agreement with ACell. S.D. Abramowitch received a research grant from the National Institutes of Health, R01 HD-061811, and has a cooperative research agreement with ACell. 
Conclusions-Parity has a negative impact on the mechanical properties of the ewe vagina, as also observed in the NHP. The ewe may serve as an alternative model for studying parity and ultimately prolapse development.

\section{Keywords}

Ewe; Mechanical properties; Pregnancy; Prolapse; Vagina

\section{Introduction}

Pelvic floor disorders (PFDs) often have a negative impact on a woman's quality of life and self-image [1,2]. Pelvic organ prolapse is one of the most common PFDs, with a higher incidence observed in elderly and parous women relative to young and nulliparous women [3-5]. Prolapse is defined as the loss of vaginal support to the pelvic organs, resulting in their descent into the vaginal canal $[4,6]$. Twelve percent of women in the USA undergo at least one corrective operation for prolapse by the age of 80 [7]. Although the pathogenesis of prolapse has also been linked to advancing age and obesity, [3-5, 8], parity (number of deliveries) has been identified as the leading risk factor [3-5]. Parous women are four times more likely than nulliparous women to develop prolapse after a single vaginal delivery, and this risk nearly doubles after a second delivery [4]. Interestingly, symptomatic prolapse often develops decades after a woman's last birth, suggesting that further research is needed to understand the mechanisms associated with vaginal delivery that lead to prolapse development.

Vaginal delivery is mechanically demanding, as it requires two competing processes:

1. Very low stiffness of the vagina and its supportive tissues to afford maximal distension during delivery

2. Maintenance of vaginal tensile strength to avoid injury.

Using imaging and computational modeling, it has been estimated that muscles in the human pelvic floor stretch 2-3 times their initial length during vaginal delivery $[9,10]$.

Unfortunately, owing to the ethical dilemmas surrounding the procurement of human vaginal tissue and the limitations with studying vaginal delivery in humans, knowledge regarding the impact of pregnancy and vaginal delivery on the vagina and surrounding pelvic muscles and connective tissues is limited. Therefore, animal models are crucial for expanding this knowledge.

The rodent and nonhuman primate (NHP) are commonly used to investigate changes in the vagina with pregnancy and vaginal delivery [11-15]; however, these models have limitations. The small size of rodents, along with their posture and small pup size relative to their vaginal diameter make maternal birth injury during vaginal delivery and subsequent prolapse development unlikely [16]. The NHP does sustain maternal birth injuries as a result of vaginal delivery and naturally develops prolapse [17]. However, the limited availability and expense of using NHPs makes conducting large and long-term studies difficult. Ewes, however, may serve as good alternatives as they are relatively inexpensive compared with NHPs, sustain maternal birth injuries, and develop prolapse naturally [18-20]. Additionally, 
the pelvic connective tissue of the ewe has three primary levels of support, similar to that of humans, and the ewe is considered an acceptable model for studying human reproduction $[16,21]$. Yet, the appropriateness of using the ewe as a model for studying the impact of pregnancy and delivery on the vagina is currently not clear.

Previously, it has been shown using the rodent model that the vagina and its supportive connective tissues undergo profound maternal adaptations in preparation for delivery. These maternal adaptations include decreased collagen fiber compaction and increased vaginal distensibility [11, 12]. Interestingly, there was no difference in the mechanical properties between the nulliparous and parous rodent vagina, demonstrating the near absence of maternal birth injury and a remarkable capacity for complete recovery postpartum [14]. This is not the case for the NHP, as the parous NHP vagina is mechanically inferior to the nulliparous vagina, which is indicative of permanent damage and long-term changes in the vagina postpartum [13]. Since ewes are known to suffer vaginal injury during delivery and prolapse related to birth, we hypothesize that the mechanical properties of the vagina, specifically tangent modulus and tensile strength, of parous ewes might be inferior to that of nulliparous ewes, with differences on par with those observed between nulliparous and parous NHPs. Given that collagen is the primary load-bearing protein in the body, we also hypothesize that the inferior mechanical properties will be associated with a change in collagen, specifically decreased total collagen content and decreased thick collagen fibers. To test these hypotheses, the uniaxial mechanical properties, total collagen content, and collagen structure of nulliparous and parous ewe vaginas were compared. Additionally, the mechanical data on the ewe vagina were compared with previously published data from the NHP and rodent models to determine the appropriateness of the ewe as an alternative model for studying vaginal mechanics as a function of delivery and pregnancy.

\section{Materials and methods}

\section{Animals}

Fifteen female mixed breed Dorset ewes (Ovis aries) aged 2.5 to 3.5 years were utilized in this study in accordance with the University of Pittsburgh Institutional Animal Care and Use Committee (IACUC \#13051885). All animals were allowed access to food and water ad libitum and were housed with a 12-h alternating light-dark cycle. Animals were categorized into two groups, nulliparous $(n=7)$ and parous $(n=8)$. All parous animals had undergone a vaginal delivery at least 1 year previously (parity $=1$ ); data on whether the pregnancy resulted in a singleton or multiples were not available. Additionally, the weight, age, and tail length after tail docking were collected. Ewes naturally have a long tail that is docked for hygienic purposes. It has been shown that shorter tails are associated with an increased risk of rectal prolapse in ewe lambs [22]. This is likely due to a direct insult to the innervation and/or muscles supporting the rectum. Thus, differences in tail length were assessed.

A Pelvic Organ Prolapse Quantification (POP-Q) examination was performed following euthanasia, with the ewe in the dorsal supine position. All measurements were made using an approach similar to that used in humans [23]. Following the POP-Q, the reproductive tract, including the vagina, its supportive tissues, and the bladder, were removed en bloc via a transabdominal approach. The vagina was then isolated and divided into anterior and 
posterior portions. To obtain appropriately sized samples and to avoid any confounding factors caused by the urethra, samples for histology and biochemistry were taken from the anterior portion of the vagina and for biomechanical testing samples were taken from the posterior portion.

\section{Trichrome staining}

Masson's Trichrome staining was used to qualitatively confirm that vaginal samples were of full thickness and to assess gross morphological differences in the sub-layers of the vagina between the two groups. Fresh samples from the mid-region of the anterior portion of the vagina were embedded and frozen in optimal cutting temperature compound (OCT; Sakura, Tokyo, Japan), sectioned at $7 \mu \mathrm{m}$, and stained with hematoxylin and trichrome stain AB (both Sigma-Aldrich, St Louis, MO, USA). Images were taken at $\times 100$ on a Nikon Eclipse 90i imaging microscope (Melville, NY, USA) and examined by a blinded researcher for gross differences in sub-layers between the two groups.

\section{Total collagen content}

The total amount of collagen in the mid-region of the anterior portion of the vaginal samples was measured using the hydroxyproline assay as previously described [24]. Briefly, frozen samples were dried, weighed, and digested in a $125-\mu \mathrm{g} / \mathrm{ml}$ papain solution at $60{ }^{\circ} \mathrm{C}$ overnight. As a standard control, purified hydroxyproline solution $(0-16 \mu \mathrm{g})$ was prepared. Vaginal samples and aliquots of the standard were hydrolyzed in $6 \mathrm{M} \mathrm{HCl}$ at $120{ }^{\circ} \mathrm{C}$ overnight. Chloramine-T reagent (Sigma) was then added to continue the oxidation of the hydrolytes. Following oxidation, Ehrlich's aldehyde reagent (Sigma) was added to each sample, and the chromophore was developed by incubating the samples for $20 \mathrm{~min}$ at $65^{\circ} \mathrm{C}$. A spectrophotometer was then used to measure the absorbance of each sample at $550 \mathrm{~nm}$, read in triplicate. The total amount of collagen was determined by assuming that $14 \%$ of collagen is made up of hydroxyproline [24, 25]. This amount was then normalized to tissue dry weight.

\section{Collagen structure}

Collagen structure, specifically the fiber thickness, was assessed using picrosirius red staining. Full-thickness vaginal tissue samples were obtained from the anterior portion of the mid-vagina of 6 nulliparous and 6 parous animals. Samples were then oriented along the longitudinal axis of the vagina, embedded in OCT, and frozen in liquid nitrogen. Blocks of tissue were then sectioned ( $7 \mu \mathrm{m}$ thick) and stained with picrosirius red. Five images per sample were obtained at $\times 200$ in the vaginal subepithelium using circularly polarized light imaged with a Nikon Eclipse 90i imaging microscope (Melville, NY, USA). Custom Matlab software (The Mathworks, Natick, MA, USA) was used to separate the collagen fibers by hue and to quantify the collagen fiber area using a method previously described [26]. Collagen fibers were separated into four colors, which indicate fiber thickness. Colors, in order of increasing thickness, included green, yellow, orange, and red. The collagen fiber area was calculated as a percentage of the total area of the image under analysis. Finally, the collagen fiber area (per color) was divided by the total area of collagen fibers to determine the percentage of each fiber thickness based on color area (i.e. the percentage of red, orange,

Int Urogynecol J. Author manuscript; available in PMC 2016 December 30. 
yellow, and green collagen fibers relative to the total amount of collagen fibers within each image).

\section{Biomechanics}

Following excision, the posterior vagina was isolated and stored in saline-soaked gauze at $-20{ }^{\circ} \mathrm{C}$ until the day of testing $[27,28]$. On the day of testing, specimens were allowed to thaw at room temperature and tissue samples of approximately $26 \times 5 \mathrm{~mm}$ were excised from the mid-region of the vagina, longitudinally oriented. The longitudinal direction was chosen for this study, as this direction likely plays a greater role in providing support to the pelvic organs for activities of daily living outside of intercourse and childbirth. With the ends of the specimen secured by soft-tissue clamps, specimens were cut into a dog-boned shape with an aspect ratio (length to width) of at least 5 (Supplementary Material, Fig. 1). The crosssectional area (CSA) of each dog-bone-shaped specimen was measured in three areas along the mid-substance using a noncontact laser micrometer system and averaged [29]. Specimens were hydrated throughout the preparation process using $0.9 \%$ saline solution.

Before mechanical testing, two contrast markers were placed in the mid-substance region on the luminal surface of the vaginal specimens using a minimal amount of cyanoacrylate (Supplementary Material, Fig. 1). A camera (Cannon Rebel EOS T3) was used to record the marker motion and a tracking algorithm written in Mathematica (V9; Wolfram, Champaign, IL, USA) was used to capture the position of the markers throughout testing to calculate strain. For testing, specimens were aligned along the longitudinal axis of the vagina with one clamp attached to the base of an Instron ${ }^{\mathrm{TM}}$ testing machine (Version 5565; Instron Industrial Products, Grove City, PA, USA) and the other attached to the crosshead of the machine through a 50-lb load cell (Model 31; Honeywell, Morristown, NJ, USA) with $0.1 \mathrm{~N}$ resolution. Specimens were then submerged in a slackened state within a $37{ }^{\circ} \mathrm{C}$ physiological saline bath and allowed to equilibrate for approximately $30 \mathrm{~min}$ before testing. For uniaxial tensile testing, a $0.1 \mathrm{~N}$ preload (loading rate of $10 \mathrm{~mm} / \mathrm{min}$ ) was applied to establish an initial clamp-to-clamp distance and an initial inter-marker distance. This was followed by ten cycles of preconditioning to $7 \%$ of the initial clamp-to-clamp distance (roughly $4 \%$ mid-substance strain) loaded at $10 \mathrm{~mm} / \mathrm{min}$. Specimens were then loaded to failure at a rate of $10 \mathrm{~mm} / \mathrm{min}$. The load (Newtons) and elongation (millimeters) were recorded and used to generate a load-elongation curve. The measured load was then divided by the cross-sectional area and plotted versus the measured mid-substance strain recorded based on the change in inter-marker distance divided by the initial inter-marker distance. From these stress-strain curves, the tangent modulus, tensile strength, ultimate strain, and strain-energy density were calculated and used to describe the mechanical properties of the vaginal specimens $[13,14]$.

The material stiffness of each specimen, described by the tangent modulus, represents the maximum slope of the stress-strain curve calculated over a moving window of $1 \%$ strain. The tensile strength corresponds to the maximum stress that the specimen withstood before failure where stress is defined as the force divided by the un-deformed CSA of the specimen. Ultimate strain is the maximum amount of deformation that the specimen can withstand to failure and is calculated by taking the ratio of the change in length of the marker position 
relative to the initial marker position. Last, the strain-energy density was determined by calculating the area underneath the stress-strain curve to failure and is representative of the "toughness" of each specimen.

Previously, the mechanical properties of the rodent and NHP vaginas were determined using the same methods as those described above [13, 14]. For the parous animals, the rodent had a parity of at least one (the exact parity is unknown) and the median parity of the NHP was 7. Dog-boned shape specimens were created from the posterior portion of the vaginas (aspect ratio of at least 5) and the CSA was measured as described above. Additionally, contrast markers were used to track tissue deformation and specimens were tested in a heated saline bath. The tensile protocols utilized were nearly identical to those performed for the ewe with the exception that the preload for the NHP was $0.5 \mathrm{~N}$, slightly higher. A higher preload was needed to remove slack from the relatively thicker NHP vagina. All mechanical properties were defined and calculated as described previously. Given the consistency in these methods, the mechanical properties obtained for all species could be compared.

\section{Statistics}

Based on historical NHP data, a total of 5 animals per group were needed to detect at least a $25 \%$ difference between nulliparous and parous vaginal mechanical properties for a power of $80 \%$ and significance level of $p<0.05$. Kolmogorov-Smirnov tests were used to determine normality. Normally distributed data were compared using Independent sample $t$ tests and represented as mean \pm standard deviation. Non-normally distributed data were compared using Mann-Whitney $U$ tests and represented as median (interquartile range). The mechanical properties of nulliparous and parous animal models were compared using ANOVA (Gabriel's pairwise post-hoc test) or the Welch alternative for the F-ratio (GamesHowell post-hoc test) where appropriate. Statistical analysis was performed using SPSS 22.0 statistical software (IBM, Armonk, NY, USA).

\section{Results}

The demographic data of all 15 animals are shown in Table 1 . The nulliparous animals were significantly heavier than the parous animals $(p<0.001)$. Using a post-hoc linear regression analysis (data not shown), it was determined that weight did not significantly predict the changes in the mechanical properties reported below. No significant difference in tail length, genital hiatus, or total vaginal length was found between the two groups. The perineal body was significantly shorter in the parous compared with the nulliparous ewes $(p=0.001)$.

Additionally, greater anterior and posterior vaginal wall descent was observed in the parous ewes relative to nulliparous ewes, as confirmed with POP-Q points Aa, Ba, Ap, and Bp. However, no significant differences were found in apical support (points $C$ and $D$ ).

Trichrome staining confirmed that all specimens were from full-thickness vagina (Fig. 1). The ewe vagina consists of four layers: the epithelium, the subepithelium (contains the majority of the collagen matrix of the vaginal cross-section), the muscularis (contains circumferential and longitudinally oriented smooth muscle fibers), and the adventitia (contains primarily loose connective tissue). Based on gross observation, it did appear that the subepithelium in the parous animals was thinner than that of the nulliparous animals. 
The parous ewes had a greater amount of total collagen than the nulliparous ewes $(90.3 \pm 8.5$ and $83.7 \pm 11.2 \%$ respectively); however, this difference was not significant $(p=0.217)$. Overall, the distributions of collagen fiber thicknesses in the nulliparous and parous ewes were comparable, with no significant differences detected between the two groups (Supplementary Material, Table 1, Fig. 2).

All stress-strain curves exhibited the typical nonlinear shape for soft tissues, with the characteristic toe, linear, and failure regions (Fig. 2). There was no difference in the CSA of the vaginal specimens ( $p=0.190$, Table 2$)$. All failures occurred in the mid-substance of the specimen allowing for the tensile strength, ultimate strain, and strain-energy density to be reported. The tangent modulus of the nulliparous ewe vagina was approximately $51 \%$ higher than that of the parous vagina $(p=0.021)$. In addition, the nulliparous vagina was $36 \%$ stronger than the parous vagina $(p=0.021)$. In comparison to the nulliparous vagina, the parous vagina also experienced $42 \%$ more deformation $(p=0.015)$. Given these differences, the strain-energy density of the two groups was not significantly different ( $p=$ $0.660)$.

Relative to the nulliparous NHP and rodent vaginas, the tangent modulus of the nulliparous ewe was higher, but this was not statistically significant $(p=0.290)$. However, the tensile strength $(p=0.002)$, ultimate strain $(p=0.003)$, and strain-energy density $(p=0.007)$ were all significantly higher for the nulliparous ewe vagina. Specifically, the ewe was $\sim 21 \%$ stronger than the NHP $(p=0.021)$ and $27 \%$ stronger than the rodent $(p=0.002)$. The ewe vagina was also found to deform $37 \%$ more than the rodent vagina $(p=0.003)$. Last, the ewe vagina was $55 \%(p=0.030)$ and $70 \%(p=0.007)$ tougher than the NHP and rodent respectively (Table 3$)$.

Mechanical properties reported among the three animal models for the parous vagina became increasingly different (Table 4 ). The tangent modulus for the ewe was nearly $44 \%$ less than that of the rodent $(p=0.042)$, and the tensile strength was $66 \%$ higher than that of the NHP $(p=0.012)$. The parous ewe vagina deformed significantly more than both the NHP and rodent ( $p=0.002$ and $p=0.025$ respectively). Furthermore, the ewe vagina was approximately $83 \%$ tougher, relative to the NHP $(p=0.001)$, with no significant difference in toughness between the ewe and rodent vaginas.

Notably, there was a decrease in the stiffness and strength of the ewe and NHP parous vaginas relative to the nulliparous vaginas. However, this difference was not observed in the rodent. Additionally, the ultimate strain increased for the ewe and rodent parous vaginas, relative to the nulliparous vaginas.

\section{Discussion}

This study was aimed at assessing the potential use of the ewe as a model for understanding the pathogenesis of prolapse by examining the impact of parity on the mechanical properties of the vagina. Consistent with our hypothesis, the main finding of this study was that the nulliparous ewe vagina was stiffer and stronger than the parous ewe vagina. Interestingly, this result was consistent with the trends observed in our collagen analyses, although the 
latter were not significantly different. In a similar study conducted by Ulrich et al. [30], the nulliparous ewe vagina was also found to be stiffer and stronger than the parous ewe vagina, though not statistically significant. The results of our study support this finding and show significant differences between the two groups in terms of stiffness and strength. Additionally, Ulrich et al. [30] determined that the total amount of collagen in the parous ewe vagina was significantly greater than in the nulliparous ewe vagina. Our results are congruent with this finding; however, our results were not statistically different.

The second major finding of this study was that there are some differences in mechanical properties between nulliparous and parous vaginas across species, with the relative differences between nulliparous and parous ewe vaginas mirroring those in the NHP. This same change is not present in the rodent and is likely attributed to the rodent not sustaining significant maternal injury during delivery because of its small pup size relative to its vaginal diameter, and its superior capacity for recovery. The ewe and the NHP, on the other hand, are known to often sustain significant maternal injury as a result of delivery. Although not a primary outcome measure, the perineal body of the nulliparous ewe was found to be longer than that of the parous ewe. This may be a consequence of vaginal delivery resulting in a thinning of the perineal body; however, without knowing the length of the perineal body before delivery, it cannot be concluded that parity is the sole contributor to this difference.

As stated above, there were no significant changes observed with collagen analyses despite trends that were consistent with the results from mechanical testing. This may be a result of the inability of the hydroxyproline assay to detect small differences in the total amount of collagen or differences in collagen subtypes. It is known that increasing the relative ratios of the fibrillar collagens (types III and/or V to type I) can result in tissue with inferior mechanical properties, as observed in the parous ewe [16]. As this result could also be explained by other factors, including differences in collagen alignment and crosslinking, further studies examining the compositional and structural changes of the ewe vagina resulting from parity are warranted. Nevertheless, these data demonstrate that an equivalent or even higher amount of collagen does not necessarily correspond to what is observed mechanically. Since the quality of collagen is just as, if not more, critical than the amount of collagen in terms of the corresponding mechanical behavior, caution should be observed when inferring judgment about the mechanical integrity of a tissue based solely on histomorphological findings.

As anticipated, the mechanical properties of the ewe vagina do not return to virgin levels postpartum as in the rodent. The mechanical impact of parity on the ewe vagina was more congruent with that of NHPs, specifically rhesus macaques [13]. This is an interesting finding given that all ewes had a parity of 1 and the median parity for the macaques was 7. It suggests that there might be a secondary change in the multiparous vagina relative to the primiparous vagina, or that the time from the last delivery might be an important factor to consider.

Importantly, the choice of animal model is largely dependent on the research question being addressed. The uniaxial mechanical properties of the nulliparous rodent, NHP, and ewe vaginas were often statistically different, but were surprisingly similar, despite their obvious 
interspecies variations and differences in age and size (Table 3). These data suggest that, in terms of mechanics, the vagina may be comparable across species and the use of smaller and less expensive animal models may be sufficient to address research questions that are difficult and more expensive to conduct in the NHP and human. However, it should be noted that the size of the ewe and their large rumen make surgical studies logistically difficult.

Strengths of this study include the use of a large animal model that develops prolapse spontaneously and has similar vaginal tissue structure and reproduction to humans. Additionally, the mechanical protocol utilized in this study is also a major strength. This protocol is the same protocol used to assess vaginal mechanics in nulliparous and parous NHPs and rodents, thus making a comparison of the impact of parity across species possible. However, the results in this study should be interpreted considering the following limitations. First, the weight of the ewes was notably different. This likely was due to random sampling and animal availability; however, weight could not predict the changes in mechanical properties reported. Second, although the parous ewes had a parity of 1 , information on whether any of the parous animals had multiples was unavailable, which is common for these animals. Delivering twins may result in more damage to the vagina than a singleton delivery, and this variable may skew the impact of parity on the ewe vaginal mechanics. Third, the mechanical properties of the vagina were analyzed along one direction. In vivo the vagina is simultaneously loaded in multiple directions. Thus, analyzing the mechanical properties of the vagina from a biaxial approach would provide a more robust evaluation of the mechanics of the vagina. Finally, it cannot definitively be stated that the changes causing the decreases in mechanical properties observed with parity are the same changes that are observed in animals with prolapse. However, it can be said that the observed decrease in the vaginal mechanical properties with parity is consistent with what one would expect in patients who develop prolapse over time.

Overall, this study demonstrates that parity has a negative impact on the mechanical properties of the ewe vagina in a similar manner to the NHP. Thus, the ewe may serve as a cheaper alternative for studying the association between parity and maternal birth injury to the vagina and the development of prolapse. However, the use of ewes for studying the pathogenesis of prolapse must be carefully considered and further research is needed.

\section{Supplementary Material}

Refer to Web version on PubMed Central for supplementary material.

\section{Acknowledgments}

We are grateful to the National Institutes of Health for funding support R01 HD-061811. This material is based upon work supported by the National Science Foundation Graduate Research Fellowship Program under Grant No. DGE-1247842. The assistance of Matthew Falcioni from the University of Pittsburgh, Department of Statistics, in statistical analysis, and Dr. Bryan N. Brown from the University of Pittsburgh, Department of Bioengineering, in examining collagen fiber thickness, is gratefully acknowledged. Additionally, we would like to thank Christopher A. Carruthers from the University of Pittsburgh, Department of Bioengineering, for providing the MatLab code.

Funding This study was funded by the National Institutes of Health R01 HD-061811. 


\section{References}

1. Barber MD, Visco AG, Wyman JF, Fantl JA, Bump RC. Sexual function in women with urinary incontinence and pelvic organ prolapse. Obstet Gynecol. 2002; 99(2):281-289. DOI: 10.1016/ S0029-7844(01)01727-6 [PubMed: 11814510]

2. Jelovsek JE, Barber MD. Women seeking treatment for advanced pelvic organ prolapse have decreased body image and quality of life. Am J Obstet Gynecol. 2006; 194(5):1455-1461. DOI: 10.1016/j.ajog.2006.01.060 [PubMed: 16647928]

3. MacLennan AH, Taylor AW, Wilson DH, Wilson D. The prevalence of pelvic floor disorders and their relationship to gender, age, parity and mode of delivery. Br J Obstet Gynaecol. 2000; 107(12): 1460-1470.

4. Mant J, Painter R, Vessey M. Epidemiology of genital prolapse: observations from the Oxford Family Planning Association Study. Br J Obstet Gynaecol. 1997; 104(5):579-585. [PubMed: 9166201]

5. Swift SE. The distribution of pelvic organ support in a population of female subjects seen for routine gynecologic health care. Am J Obstet Gynecol. 2000; 183(2):277-285. [PubMed: 10942459]

6. Olsen AL, Smith VJ, Bergstrom JO, Colling JC, Clark AL. Epidemiology of surgically managed pelvic organ prolapse and urinary incontinence. Obstet Gynecol. 1997; 89(4):501-506. [PubMed: 9083302]

7. Wu JM, Matthews CA, Conover MM, Pate V, Jonsson Funk M. Lifetime risk of stress urinary incontinence or pelvic organ prolapse surgery. Obstet Gynecol. 2014; 123(6):1201-1206. [PubMed: 24807341]

8. Moalli PA, Jones Ivy S, Meyn LA, Zyczynski HM. Risk factors associated with pelvic floor disorders in women undergoing surgical repair. Obstet Gynecol. 2003; 101(5):869-874. [PubMed: 12738142]

9. Ashton-Miller JA, DeLancey JOL. Functional anatomy of the female pelvic floor. Ann N Y Acad Sci. 2007; 1101:266-296. [PubMed: 17416924]

10. Lien K-C, Mooney B, DeLancey JOL, Ashton-Miller JA. Levator ani muscle stretch induced by simulated vaginal birth. Obstet Gynecol. 2004; 103(1):31-40. DOI: 10.1097/01.AOG. 0000109207.22354.65 [PubMed: 14704241]

11. Alperin M, Feola A, Duerr R, Moalli P, Abramowitch S. Pregnancy-and delivery-induced biomechanical changes in rat vagina persist postpartum. Int Urogynecol J Pelvic Floor Dysfunct. 2010; 21(9):1169-1174.

12. Daucher JA, Clark KA, Stolz DB, Meyn LA, Moalli PA. Adaptations of the rat vagina in pregnancy to accommodate delivery. Obstet Gynecol. 2007; 109(1):128-135. [PubMed: 17197598]

13. Feola A, Abramowitch S, Jones K, Stein S, Moalli P. Parity negatively impacts vaginal mechanical properties and collagen structure in rhesus macaques. Am J Obstet Gynecol. 2010; 203(6):595e1595.e8.

14. Feola A, Moalli P, Alperin M, Duerr R, Gandley RE, Abramowitch S. Impact of pregnancy and vaginal delivery on the passive and active mechanics of the rat vagina. Ann Biomed Eng. 2011; 39(1):549-558. [PubMed: 20824342]

15. Lowder JL, Debes KM, Moon DK, Howden N, Abramowitch SD, Moalli PA. Biomechanical adaptations of the rat vagina and supportive tissues in pregnancy to accommodate delivery. Obstet Gynecol. 2007; 109(1):136-143. DOI: 10.1097/01.AOG.0000250472.96672.6c [PubMed: 17197599]

16. Abramowitch SD, Feola A, Jallah Z, Moalli PA. Tissue mechanics, animal models, and pelvic organ prolapse: a review. Eur J Obstet Gynecol Reprod Biol. 2009; 144(Suppl 1):S146-S158. [PubMed: 19285776]

17. Otto LN, Slayden OD, Clark AL, Brenner RM. The rhesus macaque as an animal model for pelvic organ prolapse. Am J Obstet Gynecol. 2002; 186(3):416-421. [PubMed: 11904600]

18. Ayen E, Noakes DE, Ayen E. Displacement of the tubular genital tract of the ewe during pregnancy. Vet Rec. 1997; 141(20):509-512. [PubMed: 9416674]

19. Low JC, Sutherland HK. A census of the prevalence of vaginal prolapse in sheep flocks in the Borders region of Scotland. Vet Rec. 1987; 120(24):571-575. [PubMed: 3617413] 
20. Mosdol G. Spontaneous vaginal rupture in pregnant ewes. Vet Rec. 1999; 144(2):38-41. [PubMed: 10028583]

21. Couri BM, Lenis AT, Borazjani A, Paraiso MFR, Damaser MS. Animal models of female pelvic organ prolapse: lessons learned. Expert Rev Obstet Gynecol. 2012; 7(3):249-260. DOI: 10.1586/ eog.12.24 [PubMed: 22707980]

22. Thomas DL, Waldron DF, Lowe GD, Morrical DG, Meyer HH, High RA, Berger YM, Clevenger DD, Fogle GE, Gottfredson RG, Loerch SC, McClure KE, Willingham TD, Zartman DL, Zelinsky RD. Length of docked tail and the incidence of rectal prolapse in lambs. J Anim Sci. 2003; 81(11): 2725-2732. [PubMed: 14601875]

23. Bump RC, Mattiasson A, Bo K, Brubaker LP, DeLancey JOL, Klarskov P, Shull BL, Smith ARB. The standardization of terminology of female pelvic organ prolapse and pelvic floor dysfunction. Am J Obstet Gynecol. 1996; 175(1):10-17. [PubMed: 8694033]

24. Liang R, Abramowitch S, Knight K, Palcsey S, Nolfi A, Feola A, Stein S, Moalli PA. Vaginal degeneration following implantation of synthetic mesh with increased stiffness. BJOG. 2013; 120(2):233-243. [PubMed: 23240802]

25. Neuman RE, Logan MA. The determination of hydroxyproline. J Biol Chem. 1950; 184(1):299_ 306. [PubMed: 15421999]

26. Wolf MT, Carruthers CA, Dearth CL, Crapo PM, Huber A, Burnsed OA, Londono R, Johnson SA, Daly KA, Stahl EC, Freund JM, Medberry CJ, Carey LE, Nieponice A, Amoroso NJ, Badylak SF. Polypropylene surgical mesh coated with extracellular matrix mitigates the host foreign body response. J Biomed Mater Res A. 2013; doi: 10.1002/jbm.a.34671

27. Woo SLY, Orlando CA, Camp JF, Akeson WH. Effects of postmortem storage by freezing on ligament tensile behavior. J Biomech. 1986; 19(5):399-404. [PubMed: 3733765]

28. Rubod C, Boukerrou M, Brieu M, Dubois P, Cosson M. Biomechanical properties of vaginal tissue. I. New experimental protocol. J Urol. 2007; 178(1):320-325. [PubMed: 17499792]

29. Lee TQ, Woo SLY. A new method for determining cross-sectional shape and area of soft tissues. J Biomech Eng. 1988; 110(2):110-114. [PubMed: 3379932]

30. Ulrich D, Edwards SL, Su K, White JF, Ramshaw JAM, Jenkin G, Deprest J, Rosamilia A, Werkmeister JA, Gargett CE. Influence of reproductive status on tissue composition and biomechanical properties of ovine vagina. PLoS One. 2014; 9(4):e93172. [PubMed: 24709913] 

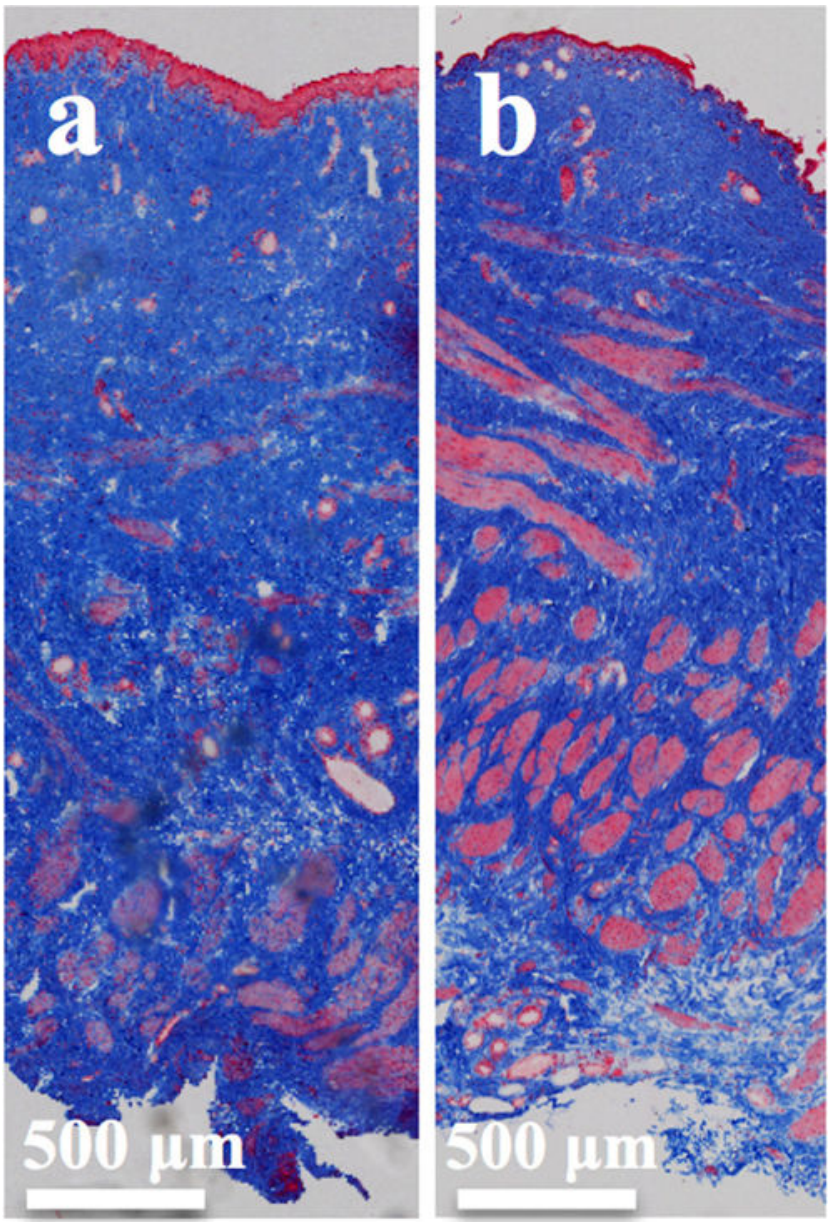

Fig. 1.

Representative trichrome images of vaginal samples from a nulliparous and $\mathbf{b}$ parous ewes. Images demonstrate that the vaginal samples are of full thickness, consisting of the epithelium (first sublayer stained pink), the subepithelium (second sub-layer stained blue), the muscularis (third sub-layer stained pink), and the adventitia (last sub-layer stained blue) 

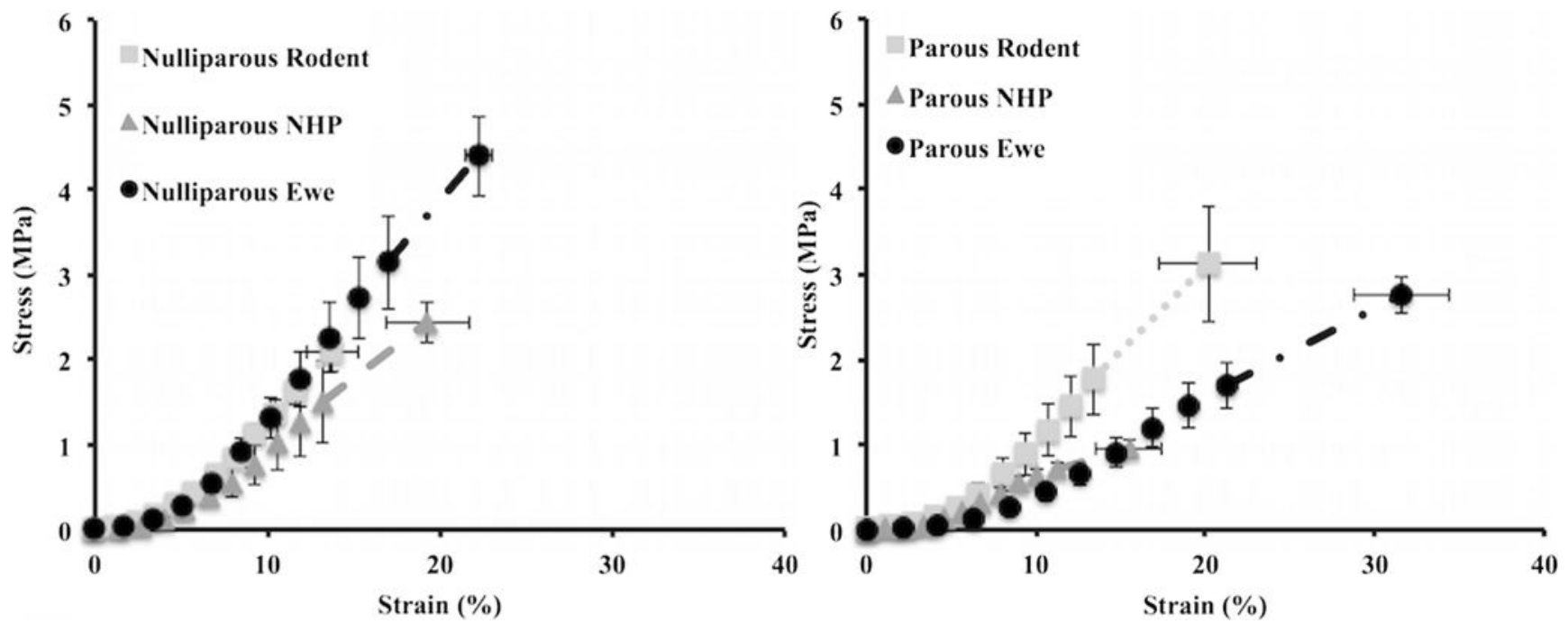

Fig. 2.

Average stress-strain curves for nulliparous and parous animals. The stress-strain curve for the nulliparous ewe had a shorter toe region and steeper slope than that of the parous ewe, resulting in significant differences in the tangent modulus, tensile strength, and ultimate strain (Table 2). Additionally, the slopes of the nulliparous animal curves were comparable. However, the slope of the parous rodent stress-strain curve was steeper than that of the parous nonhuman primate (NHP) and the parous ewe. The last data point for each curve represents the maximum average stress and the corresponding strain value observed before failure. Error bars represent the standard error of the mean (SEM). Note that SEM, instead of the standard deviation, was utilized for aesthetic purposes 


\section{Table 1}

Demographic and pelvic organ prolapse quantification scores of ewe vaginas

\begin{tabular}{|c|c|c|c|}
\hline Variable & Nulliparous $(n=7)$ & Parous $(n=8)$ & $p$ value \\
\hline Age (years) ${ }^{a}$ & $2.5-3.5$ & 2.5 & N/A \\
\hline Weight $(\mathrm{kg})^{b, c}$ & $68.3 \pm 2.6$ & $50.1 \pm 3.3$ & $<0.001^{*}$ \\
\hline Tail length $(\mathrm{cm}) b, c$ & $6.3 \pm 1.5$ & $6.3 \pm 3.0$ & $0.982^{*}$ \\
\hline Genital hiatus $(\mathrm{cm})^{d}$ & $2.0(1.0)$ & $2.0(0.5)$ & $0.36^{* *}$ \\
\hline Perineal body $(\mathrm{cm})^{d}$ & $2.5(0.5)$ & $1.8(0.5)$ & $0.001^{* * *}$ \\
\hline $\mathrm{C}(\mathrm{cm})^{d}$ & $-9(2.5)$ & $-8(2.9)$ & $0.293^{* *}$ \\
\hline $\mathrm{D}(\mathrm{cm})^{d}$ & $-10(2.5)$ & $-9.6(1.3)$ & $0.908^{* *}$ \\
\hline $\mathrm{Aa}(\mathrm{cm})^{d}$ & $-2.5(1.0)$ & $-1.5(2.1)$ & $0.038^{* * *}$ \\
\hline $\mathrm{Ba}(\mathrm{cm})^{d}$ & $-2.5(1.0)$ & $-1.5(2.1)$ & $0.038^{* * *}$ \\
\hline $\mathrm{Ap}(\mathrm{cm})^{d}$ & $-2.5(1.3)$ & $-1.0(2.4)$ & $0.034^{* * *}$ \\
\hline $\mathrm{Bp}(\mathrm{cm})^{d}$ & $-2.5(1.3)$ & $-1.0(2.4)$ & $0.034^{* *}$ \\
\hline Total vaginal length $(\mathrm{cm})^{b, c}$ & $10.6 \pm 1.6$ & $11.4 \pm 0.4$ & $0.235^{* * *}$ \\
\hline Parity & $0(0)$ & $1(0)$ & N/A \\
\hline \multicolumn{4}{|c|}{$*$ value obtained using Independent samples $t$ test } \\
\hline \multicolumn{4}{|c|}{${ }^{a}$ Ages are approximate (exact ages are unknown) } \\
\hline \multicolumn{4}{|c|}{$b_{\text {Data are represented as mean } \pm \text { standard deviation }}$} \\
\hline${ }^{c}$ Normally distributed data & & & \\
\hline
\end{tabular}




\section{Table 2}

Cross-sectional area measurements and uniaxial mechanical properties of longitudinal vaginal specimens for the ewe

\begin{tabular}{|c|c|c|c|}
\hline Variable & Nulliparous $(n=7)$ & Parous $(n=8)$ & $p$ value \\
\hline Cross-sectional area $\left(\mathrm{mm}^{2}\right)^{a, b}$ & $3.39 \pm 1.14$ & $4.76 \pm 2.39$ & $0.190^{*}$ \\
\hline Tangent modulus (MPa) $)^{a, b}$ & $34.3 \pm 13.0$ & $16.9 \pm 5.4$ & $0.021^{* *}$ \\
\hline Tensile strength (MPa) $)^{a, b}$ & $4.4 \pm 1.7$ & $2.8 \pm 0.6$ & $0.021^{*}$ \\
\hline Ultimate strain $(\%)^{a, b}$ & $22.2 \pm 2.8$ & $31.5 \pm 8.5$ & $0.015^{* *}$ \\
\hline Strain-energy density (MPa) $)^{a, b}$ & $0.4 \pm 0.2$ & $0.4 \pm 0.1$ & $0.660^{*}$ \\
\hline \multicolumn{4}{|c|}{$p$ value obtained using Independent samples $t$ test } \\
\hline \multicolumn{4}{|c|}{${ }^{* *}$ value obtained using Mann-Whitney $U$ test } \\
\hline${ }^{a}$ Data are represented as mean $\pm \mathrm{st}$ & dard deviation & & \\
\hline
\end{tabular}




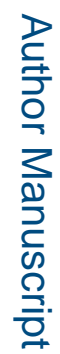

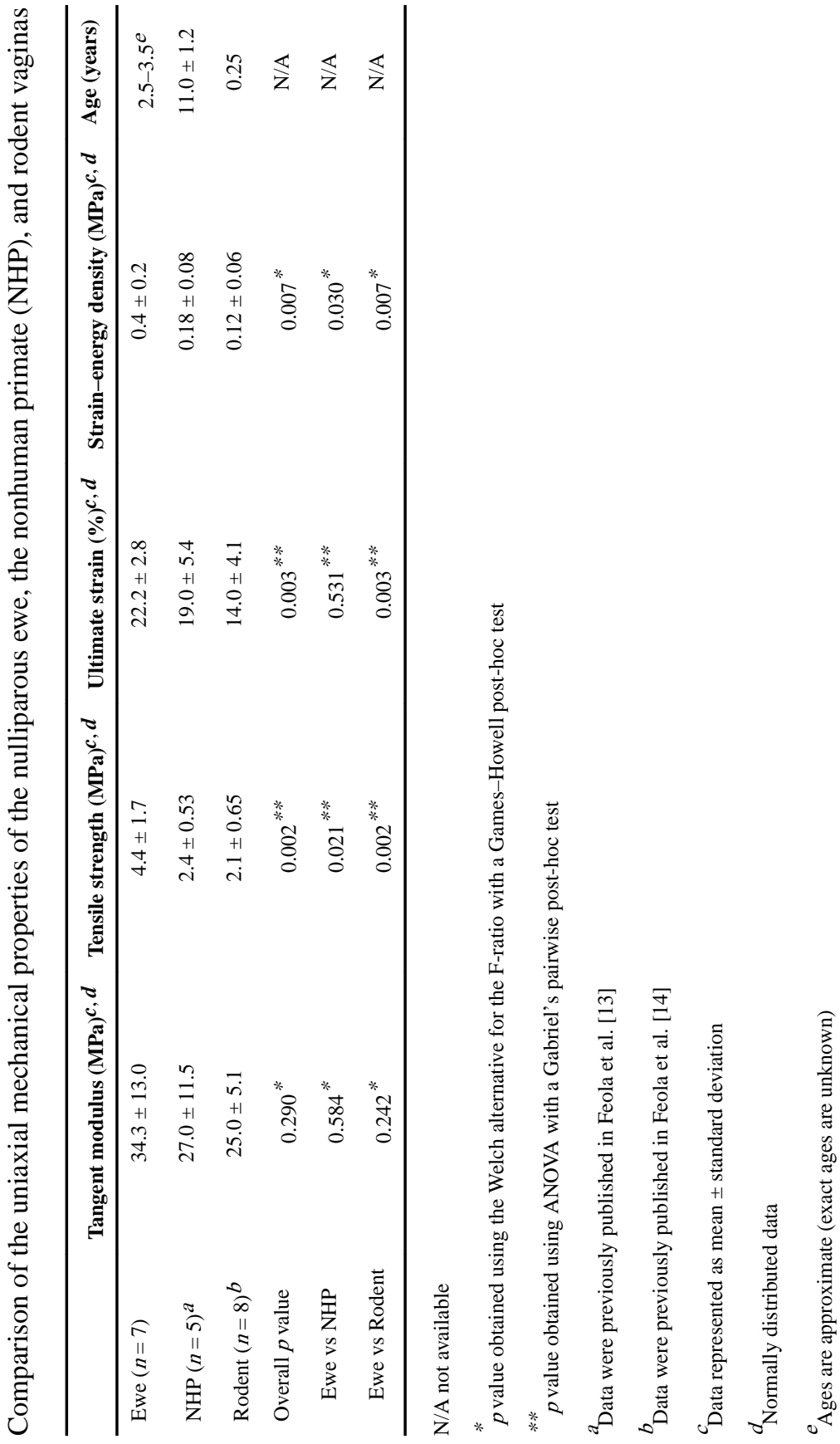




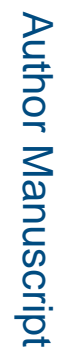

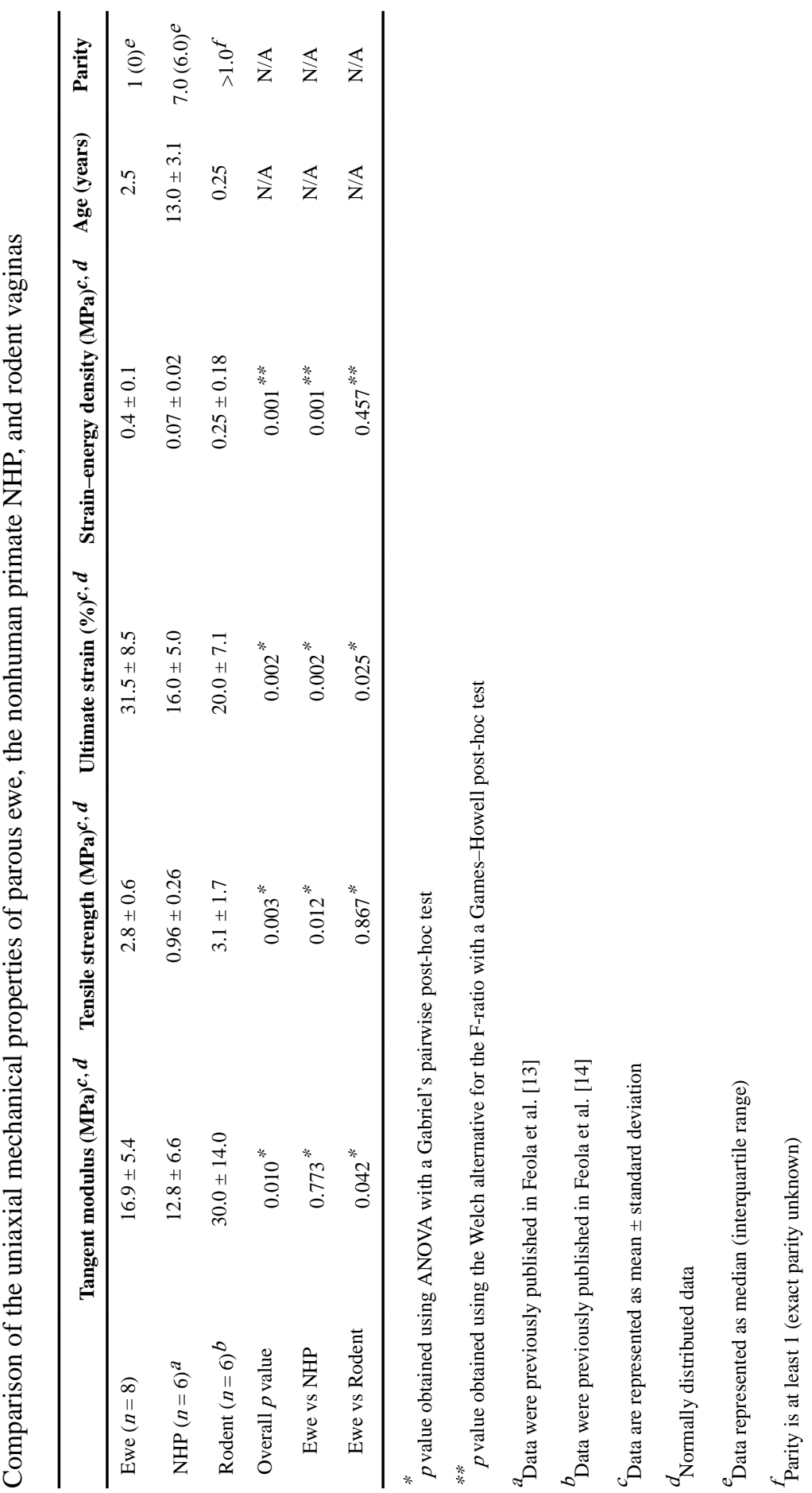

Int Urogynecol J. Author manuscript; available in PMC 2016 December 30. 